\title{
A CHALLENGING NEUROLOGICAL COMPLICATION IN A YOUNG HIV-INFECTED WOMAN
}

\author{
Irina Ianache ${ }^{1}$, Cristiana Oprea ${ }^{1,2}$ \\ 1 "Victor Babes" Clinical Hospital for Infectious and Tropical Diseases, Bucharest \\ 2 "Carol Davila" University of Medicine and Pharmacy Bucharest
}

\begin{abstract}
Background: The management of HIV infected patients with immune reconstitution inflammatory syndrome (IRIS) represents a challenge, especially in those with cerebral opportunistic infections.

Case description: We present a 26 year-old woman, diagnosed with HIV infection and admitted in our hospital with severe anemia, depression and anxiety. Lab screens showed severe anemia and immunosuppression (CD4 cell count 63 cells $/ \mathrm{mm}^{3}$ ) and high plasma HIV-RNA $\left(6.25 \log _{10}\right.$ copies $\left./ \mathrm{mL}\right)$. After one month treatment with lamivudine 3TC, tenofovir (TDF) and raltegravir RAL and important immune recovery (CD4 192 cells $/ \mathrm{mm}^{3}$ ) and significant decay in HIV viral load to $1.5 \log 10$ copies $/ \mathrm{mL}$ were noticed. The initial clinical evolution was unfavorable with the appearance of generalized seizures, movement disturbances, auditory hallucinations and right hemiparesis. CSF exam was normal with negative bacterial and fungal cultures but with detectable HIV-RNA - 82 copies $/ \mathrm{mL}\left(1.90 \log _{10}\right.$ copies $/ \mathrm{mL}$ ). Brain MRI showed hyperintense lesions on $\mathrm{T} 2$ and FLAIR sequences and hypointense on T1 sequences, localized in fronto-temporal areas, right cerebellum and pons. In evolution, there was a continuing worsening of psychiatric and neurological symptoms with severe tremor, axial hypotonia, generalized weakness and severe sleep disturbances. Atypical brain MRI lesions in the presence of severe psychiatric symptoms raised difficulties in establishing the cause of the neurological complication. The important progression of the lesion in the white matter, the appearance of contrast enhancement and the presence of JC - DNA in CSF suggested the diagnosis of PML IRIS. The patient was treated with high doses of cortisone, antipsychotic drugs and continued cART, with a slow clinical recovery and brain MRI improvement starting with 4 months of treatment.

Conclusion: The diagnosis of the neurological complication was difficult due to the atypical onset, the presence of concomitant psychiatric symptoms and the atypical initial brain imaging.
\end{abstract}

Keywords: HIV infection, psychiatric symptoms, PML IRIS

\section{BACKGROUND}

Progressive multifocal leukoencephalopathy (PML) is a severe opportunistic CNS infection caused by JC virus that occurs in immunosupressed hosts, especially in HIV-infected patients $(1,2)$. It is characterized by the demyelination of the central nervous substance with lytic infection of the oligodendrocytes (3) and it is considered to be one of the most frequent causes of mortality in HIV-infected patients with neurologic involvement (4). PML has no specific efficient treatment, but it was demonstrated that almost half of the patients with restored immunological status under cART presented also a stabilization of the cerebral lesions and of the neurological symptoms $(5,6)$.

The incidence of PML in HIV infected patients decreased in cART era associated with a significant increase in the survival rate. However, almost half of the patients still die in the first 6 months after cART initiation (3,7-9).

Immune reconstitution inflammatory syndrome (IRIS) may represent a challenge for the management of HIV-infected patients. It is characterized by a paradoxical worsening of clinical 
presentation following cART initiation (2). It has been estimated that almost a third of patients diagnosed with PML who receive cART will develop PML IRIS (9-11).

\section{CASE DESCRIPTION}

We present the case of a 26 year-old woman who was diagnosed with HIV and severe anemia in a Hematology Department and was admitted in our department in June 2015.

Her medical history was unremarkable excepting a herpetic keratitis and recurrent herpetic genital infections.

At admission in our department, the clinical exam revealed a pale patient with some mood disturbances, mild depression and anxiety. The lab screen showed severe anemia ( $\mathrm{Hb} 6,7 \mathrm{~g} / \mathrm{dl})$ and moderate thrombocytopenia. She had severe immunosuppression (CD4 cell count - 63/ $\mathrm{mm}^{3}$ ) and very high HIV plasma viral load (VL) (6.25 $\log _{10}$ copies/mL).

Anemia was corrected and combined antiretroviral treatment cART with lamivudine, tenofovir and darunavir boosted with ritonavir was initiated. After 10 days of treatment the boosted protease inhibitor (darunavir/ritonavir) was switched to integrase inhibitor (raltegravir) due to gastro-intestinal intolerance and rash. The patient was discharged and after one month on cART an important increase in the CD4 cell count to 192 cells $/ \mathrm{mm}^{3}$ and a significant decrease in HIV VL to $1.5 \log _{10}$ copies $/ \mathrm{mL}$ was noticed

At the end of July, 45 days after cART initiation, she was readmitted in our department with severe anxiety, auditory hallucinations, partial seizures with secondary generalization, movement disturbances, weakness of the right body and ataxia. The neurological deficit was progressive and after a few days she developed palsy and paresthesia of the right limbs

CSF exam was normal, with 2 cells $/ \mathrm{mm}^{3}$, proteins $0.42 \mathrm{~g} / \mathrm{l}$, negative smears and cultures for bacteria and fungi and detectable HIV-VL (1.9 $\log _{10}$ copies $/ \mathrm{mL}$ ).

The first brain MRI showed hyperintensity on T2 and FLAIR sequences and hypointensity on

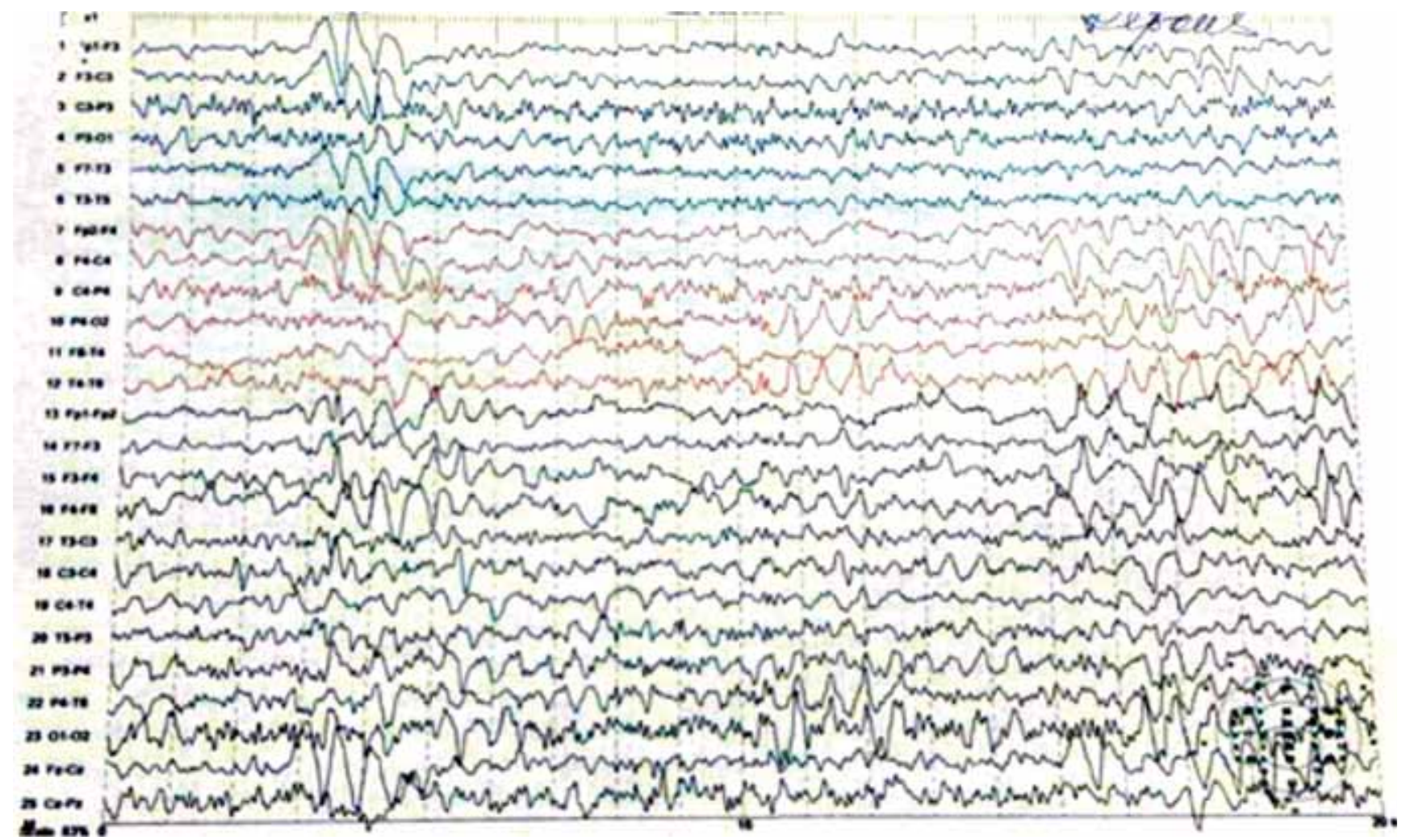

FIGURE 1. EEG showed sharp frontal delta waves. 


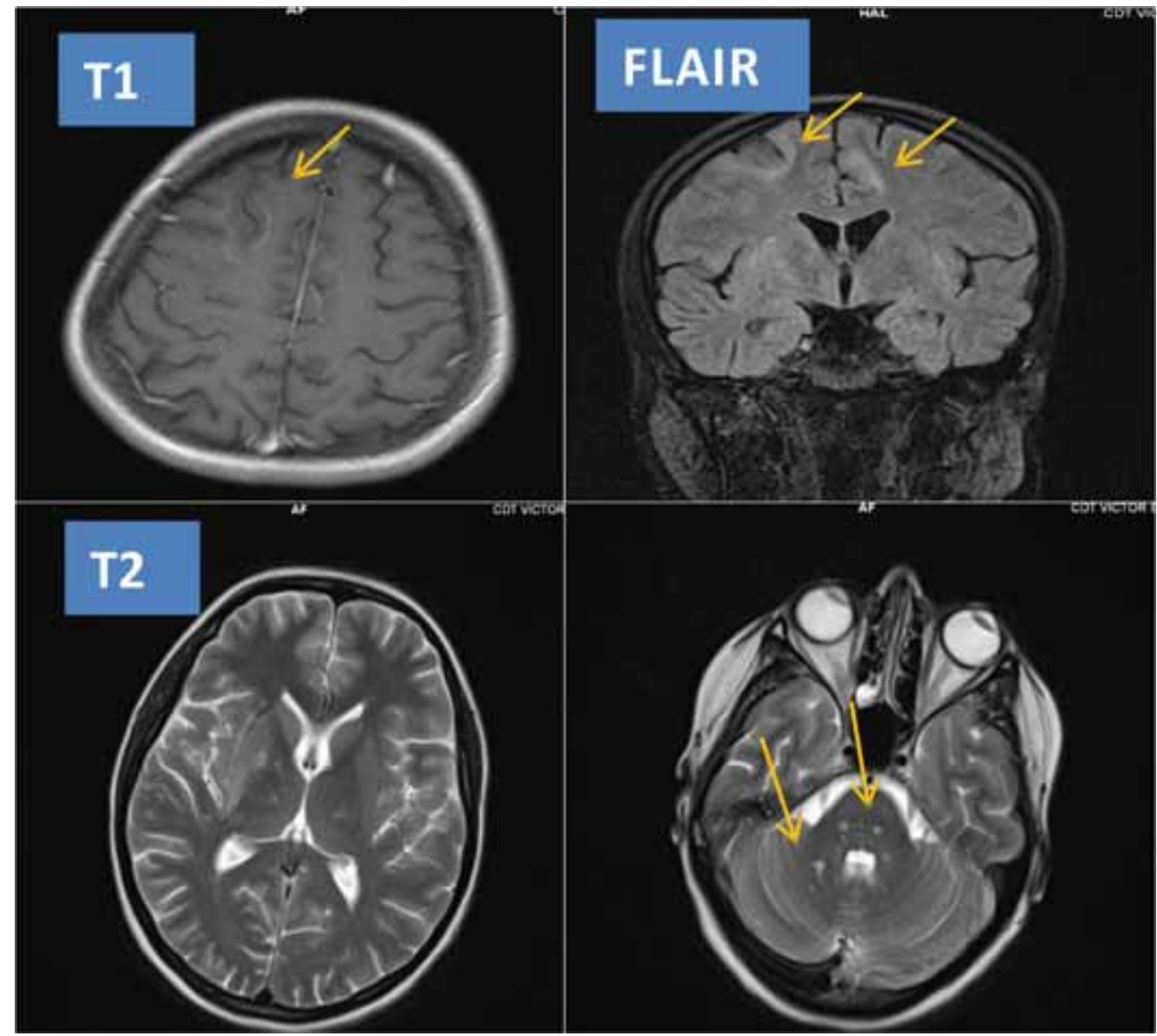

FIGURE 2. Brain MRI performed at first admission. Hyperintense lesions in T2 and FLAIR sequences and hypointense in T1, localized in the right cerebellar hemisphere and in the pons.

T1, localized in the right cerebellar hemisphere and also symmetric in the pons with a maximum of $3 \mathrm{~mm}$ in diameter. Similar signal changes were observed in the supratentorial region with important involvement of the cortex and subcortical areas, right temporal and insular areas being more affected than left insula, with extension in the frontal region, predominantly on the right side. The lesion involved also the subcortical "U" fibres and was hyperintense on FLAIR sequence. The same signal changes appeared in the left paramedian frontal cortical and subcortical areas. All these lesions were without contrast enhancement, excepting a discrete one at the edges of right-sided frontal lesions (Fig. 2).

Clinical presentations, initial negative CSF results and atypical brain MRI lesions made the diagnosis of the neurological complication more difficult.
We took into consideration two major diagnosis: viral encephalitis (HSV, CMV) and progressive multifocal leukoencephalopathy. HSV encephalitis could be suspected due to her past medical history with recurrent herpetic infections, the clinical presentation with seizures, focal motor deficit and psychiatric symptoms due to temporal lobe involvement and the MRI images with T2 and FLAIR hyperintensity in the frontotemporal region. On the other hand, there were no cingular lesions and she didn't present a chaotic delirium as described in this kind of encephalitis. However, because HSV encephalitis could not be excluded from the beginning, the patient was treated initially with injectable acyclovir (10 days), corticotherapy and antiepileptic treatment with valproate. The patient had a slight improvement of the motor deficits, but with persistence and even worsening of the psychiatric symptoms (severe anxiety, depression and insomnia). The 
HSV serology was negative, both in plasma and CSF and a negative result for PCR-DNA-HSV in CSF finally excluded this diagnosis.

CMV etiology was also excluded by the MRI images, which were not suggestive for a CMV encephalitis (usually described as hyperintense T2 lesions in the white matter, predominantly periventricular) and the negative PCR-DNACMV in CSF. PML was also considered in the differentials due to the progressive onset, in an afebrile severe immunosuppressed patient, who developed focal motor deficits and neurocognitive disorders, but the encephalitic symptoms and the atypical MRI imaging made this diagnosis less probable.

The second MRI, performed one month later, showed a progressive evolution of the supratentorial lesions, with enlarged size, signal changes and a considerably tendency to confluence, mainly in the right frontal, temporal and insular areas. In addition, axial T2 and coronal FLAIR show multiple hyperintense asymmetrical lesions extended predominantly in the subcortical white matter from the right cerebral hemisphere, left corona radiata and left paramedian frontal area. A profound subcortical

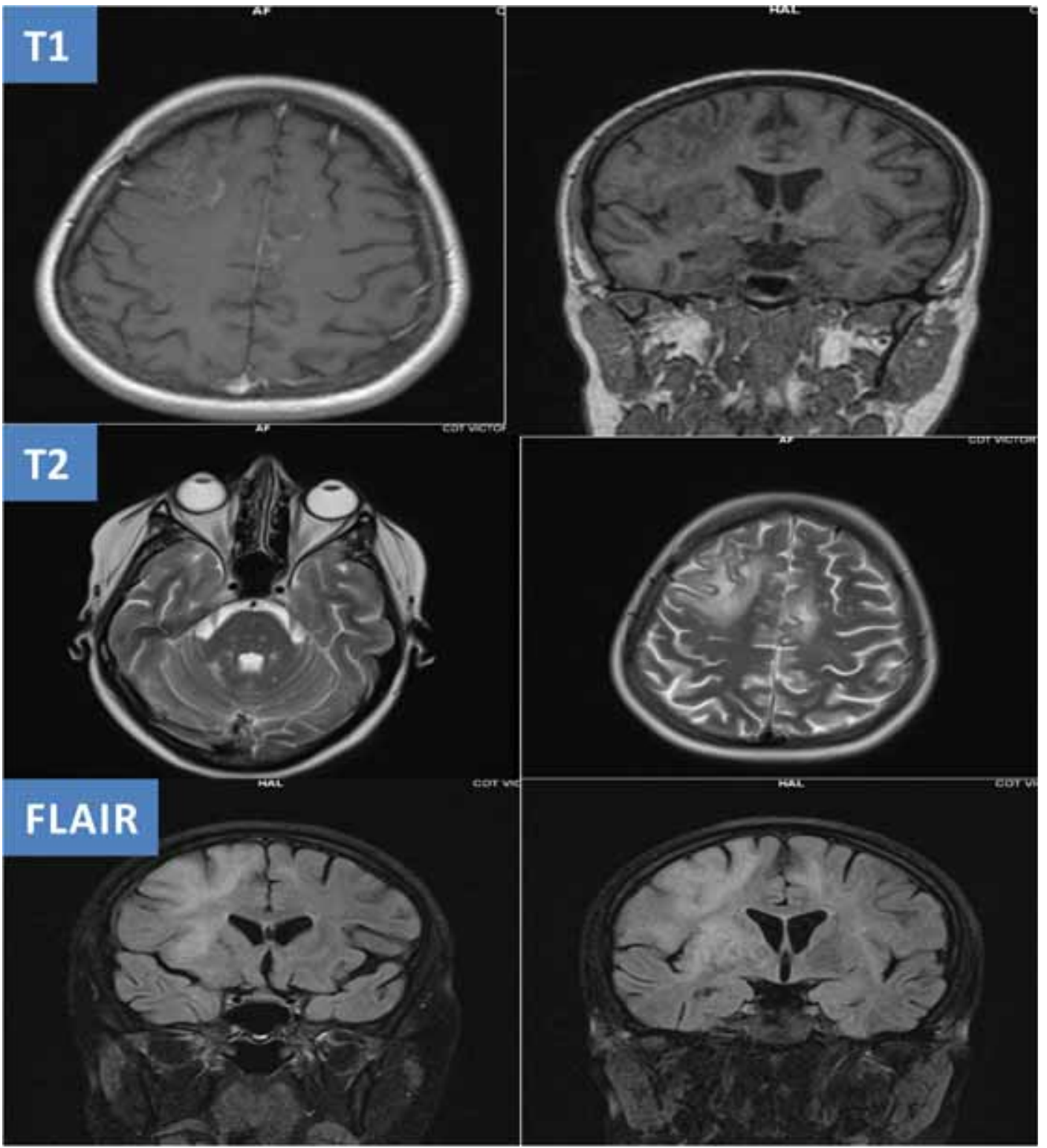

FIGURE 3. Brain MRI performed after one month on cART. Progressive evolution of the supratentorial lesions and cerebellar lesions with extension of the contrast enhancement in the frontal and parietal areas 
T1-hypointensity was noticed, associated with a progressive atrophy with tendency to cavitation and FLAIR-hyperintesity in the adjacent areas. Lesions previous described in the right cerebellar hemisphere had an increased diameter, but the brainstem lesions remained stable. An extension of the contrast enhancement in the frontal and parietal areas bilateral was observed (Fig. 3). Brain MRI performed 3 months after the cART initiation (October 2015) showed the continuous extension of the lesions, both in cerebral hemispheres and cerebellar areas with significant edema and mass effect on the right lateral ventricle and important contrast enhancement (Fig. 4).

Taking into consideration the progressive evolution of lesions previously described and the expansion of contrast enhancement, high dose corticotherapy was initiated, without interrupting cART.

The progressive aggravation of the cerebral lesions and the positive result for JC DNA in CSF raised the challenge to differentiate between classic PML and PML IRIS. PML could be suspected due to its onset and clinical presentation, but in this case, the first MRI imaging and the encephalitic symptoms were not characteristic for this etiology.

There were strong arguments to sustain IRISPML: the typical imaging for IRIS-PML on the second and third brain MRI, with extended lesions in the white matter, mass effect on the lateral ventricle and contrast enhancement. Another important argument was the increase in the

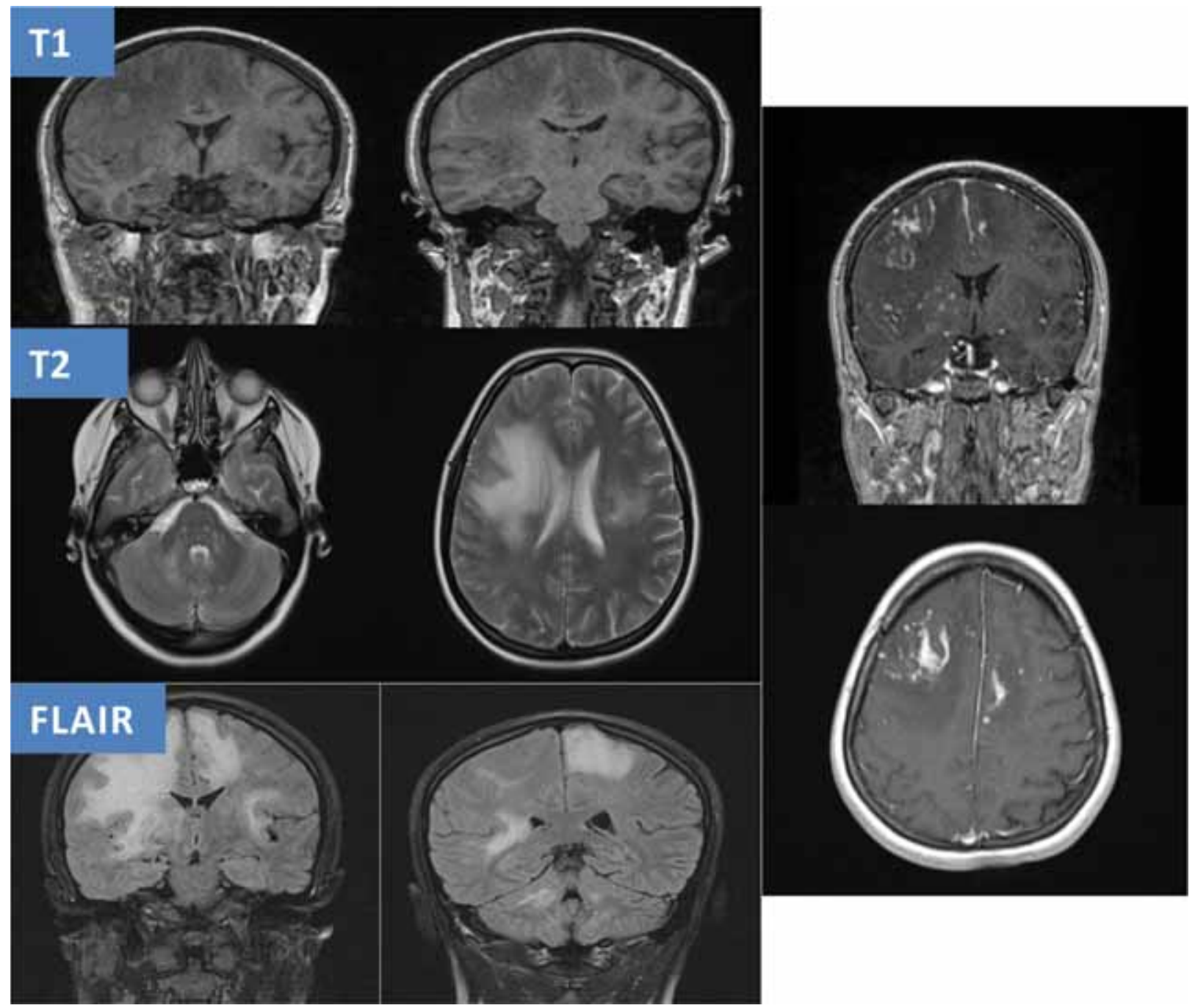

FIGURE 4. Brain MRI performed after 2 months on cART. Progressive evolution of infra and supratentorial lesions, with significant edema and mass effect on the right lateral ventricle and important contrast enhancement 
CD4 cell count, the important decay in HIV-VL (with more than 2 logs) and the rapid increase in the circulating CD8 cells. We noticed a clinical deterioration in the first months on cART, more rapid than in the classic PML and a favorable response (even if slow) to high doses of corticotherapy.

Another problem in the differentials was the presence of severe psychiatric symptoms that could suggest a simultaneous onset of an organic psychiatric disorder or could appear as a result of the atypical distribution of the JC lesions.

After 4 months of treatment (cART, corticotherapy, antipsychotic drugs and antiepileptic), we noticed an important clinical improvement, with the remission of the neurological deficits and improvement of the psychiatric symptoms, with the persistence of a slight tremor at the right upper and lower limbs.
Brain MRI performed after 4 months (November 2015) showed a favorable evolution, with important remission of the lesions previously described, with no edema, mass effect or contrast enhancement (Fig. 5). The last lab screen in November 2016 showed a good immunologic status (CD4 cell count $564 / \mathrm{mm}^{3}$ ) and undetectable HIV viral load.

\section{DISCUSSION}

We presented a particular case with PML IRIS in a HIV infected young women, with an unusual clinical onset, neurological deficits combined with important psychiatric symptoms and an atypical lesions on the initial brain MRI

The onset of the neurological symptoms after the cART initiation and the clinical deterioration after the immune recovery and the important

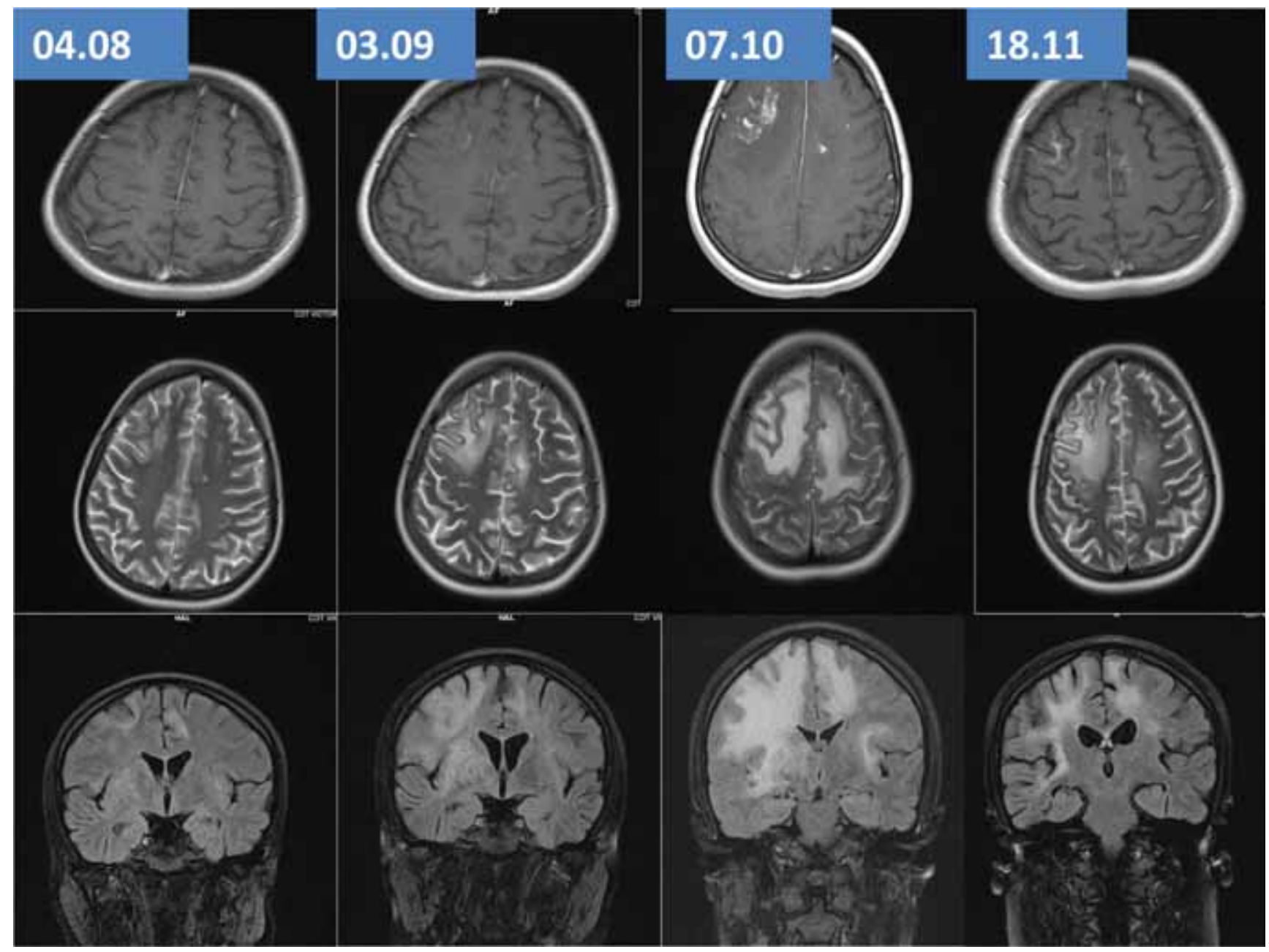

FIGURE 5. Evolution of CNS lesions on brain MRI. Significant remission of CNS lesions after 4 months on cART and corticotherapy, with no edema, mass effect or contrast enhancement 
plasma HIV-VL decrease (with more than 2 logs) after only one month of treatment, were important arguments for an unmasking PML IRIS. This type of IRIS is described when a new neurologic aggravation appears after cART initiation in a HIV-infected patient with severe immunosupression. It usually occurs between three weeks and three months of antiretroviral treatment, when the immunological recovery is considered to reach a peak $(2,12-14)$.

Interestingly, a large number of studies suggested a frequent involvement of cerebellum in patients who develop unmasking IRIS-PML, with singular lesions in the infratentorial areas or associated with cerebral lesions. More theories were proposed in order to explain this pattern, including the infection of internal neuronal layer of cerebellum cells, without affecting the oligodentrocytes or a delayed immune recovery in this area, comparing to other brain regions. In addition, this particular tropism of JC virus was frequently associated with cerebellar atrophy in HIV-infected subjects (15-17). In our case, the demyelinating lesions developed progressively in the cerebellum, in the brain stem but also in the supratentorial areas and associated contrast enhancement.

Survival was frequently associated with contrast enhancement, earlier initiation and a longer time on corticotherapy, latest studies suggesting that contrast intensity is associated with the localization of the lesions and the severity of inflammation $(9,18,19)$. It is also suggested that patients with unmasking PML IRIS have a better survival rate compared to the patients with paradoxical IRIS, who seem to have larger cerebral MRI lesions and a more rapid onset of the symptoms $(2,18)$. Contrast enhancement followed by treatment with corticosteroids seems to be a favorable predictor for survival. It is also suggested that PML IRIS is more often responsible for a higher mortality rate compared to the progressive type of classic PML (9). Even if there are limited studies and data are still missing, the association of cART with high doses of corticosteroids is the preferred option for treatment in PML IRIS $(2,18,20)$.

We faced difficulties in interpreting the cause of the concomitant severe psychiatric symptoms and a simultaneous onset of an organic psychiatric disorder couldn't be ruled out initially.

This case is singular, because we didn't found any other published data describing a patient with PML IRIS and severe psychiatric symptoms.

The association of concomitant psychiatric symptoms represented a real challenge for the management of the neurological complication in this young HIV-infected woman, diagnosed as a late-presenter.

\section{REFERENCES}

1. Tavazzi E., White K.M., Khalili K. "Progressive multifocal leukoencephalopathy: clinical and molecular aspects," Reviews in Medical Virology, vol. 22, no. 1, pp. 18-32, 2012

2. Kurukumbi M., Steiner S., Dunlap S. et al. Case Report: A Rare Case of Immune Reconstitution Inflammatory Syndrome Development in an Immunocompromised Patient with Progressive Multifocal Leukoencephalopathy and Multicentric Castleman's Disease; Hindawi Publishing Corporation Case Reports in Neurological Medicine Volume 2013, Article ID 460701

3. Gasnault J., Costagliola D., Chavez H.H. et al. Improved Survival of HIV-1-Infected Patients with Progressive Multifocal

Leukoencephalopathy Receiving Early 5-Drug Combination Antiretroviral Therapy; PLoS ONE; June 2011 Volume 6 Issue 6 e20967

4. Fanjul F., Riveiro-Barciela M., Gonzalez J. et al. Short communication: Evaluation of progressive multifocal leukoencephalopathy treatments in a Spanish cohort of HIV-infected patients: do protease inhibitors improve survival regardless of central nervous system penetration-effectiveness (CPE) score?; HIV Medicine (2013), 14, 321-325

5. Cinque P., Koralnik I.J., Gerevini S. et al. Progressive multifocal leukoencephalopathy in HIV-1 infection, The Lancet Infectious Diseases, vol. 9, no. 10, pp. 625-636, 2009 
6. Rodríguez M., Sánchez F.A.S., Rivero .CL. Case Report: Maraviroc Failed to Control Progressive Multifocal Leukoencephalopathy-Associated IRIS in a Patient with Advanced HIV Infection; Hindawi Publishing Corporation Case Reports in Medicine Volume 2014, Article ID 381480

7. d'Arminio Monforte A., Cinque P., Mocroft A. et al. Changing incidence of central nervous system diseases in the EuroSIDA cohort; Ann Neurol 2004; 55:320-328.

8. Gasnault J., Taoufik Y. New trends in progressive multifocal leukoencephalopathy. Rev Neurol. 2006, Paris. 162: 43-56

9. Post M.J.D., Thurnher M.M., Clifford D.B. et al. CNS-Immune Reconstitution Inflammatory Syndrome in the Setting of HIV Infection, Part 1: Overview and Discussion of Progressive Multifocal Leukoencephalopathy-Immune Reconstitution Inflammatory Syndrome and Cryptococcal-Immune Reconstitution Inflammatory Syndrome; AJNR Am J Neuroradiol 34:1297-307 Jul 2013

10. Tan K., Roda R., Ostrow L. et al. PML-IRIS in patients with HIV infection: clinical manifestations and treatment with steroids. Neurology 2009; 72:1458-64

11. Major E. Progressive multifocal leukoencephalopathy in patients on immunomodulatory therapies. Annu Rev Med 2010; 61:35-47

12. Cinque P., Bossolasco S., Brambilla A.M. et al. The effect of highly active antiretroviral therapy-induced immune reconstitution on development and outcome of progressive multifocal leukoencephalopathy: study of 43 cases with review of the literature, Journal of NeuroVirology, vol. 9, supplement 1, pp. 73-80, 2003.
13. Bag A.K., Cure J.K., R, Chapman P.R. et al. JC virus infection of the brain. AJNR Am J Neuroradiol 2010;31:1564-76

14. Vendrely A., Bienvenu B., Gasnault J. et al. Fulminant inflammatory leukoencephalopathy associated with HAART-induced immune restoration in AIDS-related progressive multifocal leukoencephalopathy. Acta Neuropathol 2005; 109:449-55

15. Navdeesh S., McCutchan J.A. Unmasking of PML by HAART: Unusual Clinical Features and the Role of IRIS; J Neuroimmunol. 2010 February 26; 219(1-2): 100-104

16. D'Amico R., Sarkar S., Yusuff J. et al. Immune reconstitution after potent antiretroviral therapy in AIDS patients with progressive multifocal leukoencephalopathy. Scand J Infect Dis 2007;39:347-50.

17. Du Pasquier R.A., Corey S., Margolin D.H. et al. Productive infection of cerebellar granule cell neurons by JC virus in an HIV+ individual. Neurology 2003; 61:775-82

18. Tan K., Roda R., Ostrow L. et al. PML IRIS in patients with HIV infection: cinical manifestations and treatment with steroids, Neurology, vol. 72, no. 17, pp. 1458-1464, 2009.

19. Koralnik I.J. Progressive multifocal leukoencephalopathy revisited: has the disease outgrown its name? Ann Neurol 2006;60:162-73

20. Tan C.S., Koralnik I.J. Progressive multifocal leukoencephalopathy and other disorders caused by JC virus: clinical features and pathogenesis. The Lancet Neurology, vol. 9, no. 4, pp. 425-437, 2010 